Methods We systematically identified peer-reviewed articles, published 2000-2016, which reported STI bio-specimen testing and rates among homeless persons in the US. Articles $(n=9)$ were grouped by age (e.g. youth $=5$; adults $=4$ ); STI rates and correlate factors were described by gender.

Results Across STIs, rates varied from $0.4 \%-17 \%$ among homeless youth $(\mathrm{n}=5)$, and $0.9 \%-52.5 \%$ among homeless adults $(n=4)$. Rates for chlamydia (CT) were similar for youth $(4.2 \%-11.6 \%) \%)$ and adults (4.6\%-10.8). For gonorrhoea, the highest rates for youth $(0.4 \%-9.9 \%)$ were double those for adults $(0.9 \%-4.1 \%) \%)$. STI rates also varied by age and gender. CT was highest among young females (6.45\%-11.0\%\%); followed by adult females (6.7\%); then young males $(4.73 \%-$ $4.8 \%)$. Rates of HSV-2 (5.52\%-11.85\%\%), HBV (1.42\%$17 \%)$ and trichomoniasis $(2.3 \%)$ were reported only in studies on youth. HCV varied among youth (3.77\%-12\%)\%) and adults $(9.8 \%-52.5 \% \%)$, with rates highest among homeless adult men. Composite STI rates were most often reported for adults (16.4\%-40.7\%\%), with highest rates among adult women who experienced intimate partner violence (IPV) (25.9\%-40.7\%)\%).For youth, longer periods of homelessness were associated with high-risk sexual behaviours. Peer social networks had protective and risk effects on sexual behaviour. For adults, frequency and length of homelessness, incarceration history, substance use, injection drug use (IDU), and IPV were associated with having an STI.

Conclusion STI rates among homeless persons varied by age and gender. There is a need for more complete STI data on homeless youth and adults. Such data can aid in understanding STI risk while homeless and how best to develop tailored prevention strategies.

\section{P4.95 DECLINING TRENDS IN RISK BEHAVIOURS AND HIVISTIS AMONG INJECTING DRUG USERS IN INDIA: FINDINGS FROM LARGE SCALE BIO-BEHAVIOURAL SURVEYS FROM THREE HIGH PREVALENT STATES}

Santosh Kumar Sharma. International Institute For Population Sciences, Mumbai, India

\subsection{6/sextrans-2017-053264.590}

Introduction Injecting Drug Users in India are at the duel risk of needle sharing and risky sexual behaviour, becoming more vulnerable to STI and HIV. Although injecting drug users constitute a risk group in themselves, there is also an overlap between drug addiction and those involved in sex work. Individuals who fall into both categories are therefore particularly vulnerable to HIV and are perhaps doubly stigmatised. Thus, studying the interface of injecting drug use and risky sexual behaviour is important to curb the pace of STI/HIV epidemic among IDUs.

Methods Data were obtained from two rounds of integrated biological and behavioural assessments (IBBA) conducted in 2006-07 and 2009-10 where 2,075 IDUs in round-1 and 1977 in round-2 were sampled. Data from both rounds were pooled. Bivariate and multivariate analysis were used for the study.

Results Data from 4052 IDUs from 6 districts of Manipur, Nagaland, and Maharashtra, were analysed. The mean age of IDUs in round- 1 was 26 years and 29 years in round-2. The majority of the IDUs were illiterate, unmarried, employed in two consecutive rounds. IDUs's duration between first drug use to first injecting drug use $\geq 2$ years were 14 times higher during round-2. HIV and any STI symptoms in the round-2 show the declining pattern. IDUs having the main regular partner and shared needle/syringe were less likely to consistency in condom use with different type of female partners across the rounds. Logistics regression analysis of experienced any STI symptom in the past 1 year indicate that IDUs, literate $(\mathrm{AOR}=1.878, \mathrm{p}<0.10)$, age at first injecting drugs 26 and above $(\mathrm{AOR}=2.192, \mathrm{p}<0.05)$ and sharing needle/syringes in the past month $(\mathrm{AOR}=2.218, \mathrm{p}<0.01)$ were significantly more likely than their counterparts. IDUs, $\geq 30$ years (15.951, $\mathrm{p}<0.01)$, literate $(3.415, \mathrm{p}<0.01)$ and shared needle/syringes in the past one month were significantly more likely to have HIV.

Conclusions Harm reduction and needle exchange program should also focus on the risky behaviour of injecting drug users through peer-based approach.

\section{P4.96 STIGMATISED BEHAVIOURAL ASPECTS TOWARDS MSM DIRECTING PERVASIVE NATURE OF ALCOHOL USE IN ANDHRA PRADESH, INDIA}

Santosh Kumar Sharma. International Institute For Population Sciences, Mumbai, India

\subsection{6/sextrans-2017-053264.591}

Introduction Men who have sex with men (MSM) in India are a core risk group for HIV. Heavy alcohol consumption is associated with increased sexual risk-taking behaviours in many cultures, in particular among MSM. This study illustrates the prevalence and correlates of Alcohol use among MSM by their sociodemographic, sexual risk and other characteristics in Andhra Pradesh, India.

Methods Data from a Behavioural Tracking Survey 2014 conducted with key populations MSM in undivided Andhra Pradesh state in India was used. The survey was conducted in the three districts (Warangal, Karim Nagar, and Nalgonda). A stratified random sampling method used to select the MSM for the survey. The total 1200 MSM were selected for the study. Descriptive statistics (proportions) and bivariate analysis were used to describe the strength and association of MSM' socio-demographic/behavioural characteristics and the dependent variable. For the bivariate analysis, the p-value was calculated by using the chi-square test. Binary logistics regression was used to determine the factor associated with ever consumption of alcohol among MSM.

Results In Andhra Pradesh, of the 1200 MSM who were interviewed, around 69\% of MSM had ever used alcohol. Approximately 31 percent MSM reported that they had experienced discrimination at public places and 11.2 percent of said that they are mentally depressed. There is the significant association between ever used of alcohol and sociodemographic, sexual risk and other characteristic $(p<0.05)$. Multivariate logistics regression demonstrated that currently married $(\mathrm{AOR}=1.6 \mathrm{p}<0.05)$, Panthi MSM (AOR1.7 $\mathrm{p}<0.05)$, wanted to experiment $(\mathrm{AOR}=2.0 \mathrm{p}<0.01)$, was attracted towards MSM $(A O R=2.9 \mathrm{p}<0.01)$, consistent condom use $(\mathrm{AOR}=1.7$ $\mathrm{p}<0.05$ ). High discrimination in Public places $(\mathrm{AOR}=1.4$ $\mathrm{p}<0.05)$. It is also reported that MSM who were in any depression, 2.1 times more likely to be affected by alcohol consumption.

Conclusions HIV-related prevention Programme among MSM in India should also focus on alcohol use and related problems in multilevel context. 